\title{
Die Himmelsreise Muhammeds. von
}

B. Schrieke.

$$
\begin{aligned}
& \text { Eine genaue kritische Untersuchung der } \\
& \text { Himmelfahrtserzählungen, von den ältesten } \\
& \text { Traditionen an . . w würde sehr lehrreich sein. } \\
& \text { Nöıde KE-ScHwalıy, Geschichte des Qo- } \\
& \operatorname{räns}^{2} \text { I } 136 .
\end{aligned}
$$

In den ältesten Muhammedbiographien wie in den modernen populären Maulids hat die Geschichte der Himmelsreise (mi'rādj) ihren festen Platz. Gewöhnlich wird sie mit der Nachtreise (isra $)$ nach Jerusalem verbunden. Die landläufige Auffassung ist nun, daß die Erzählung sich anknüpfend an einige Verse von Sūrah 53, nach dem Muster der aus der christlichen, jüdischen und persischen Literatur wohl bekannten ekstatischen Himmelsreisen entwickelt habe. Dies ist jedoch nicht mehr als eine Vermutung, welche in Wahrheit erst an. den Quellen nachgeprüft werden muß.

In vielen Redaktionen wird die Himmelfahrt noch mit der Erzählung von der Brusteröffnung und -reinigung verbunden. Letztere Geschichte soll aus einer buchstäblichen Erklärung von Sūrah 94, I entstanden sein. Auch diese Behauptung hätte man beweisen müssen, wie man auch niemals das "Warum? " der Verbindung von mi'rädj und Brusteröffnung aufzuhellen versucht hat. Der gewöhnlichen Auffassung stehen ja viele Schwierigkeiten gegenüber. Jene buchstäbliche Auslegung hätte nicht in Tabarī's Tafsìr und Buchārī fehlen dürfen. : Diese kennen sie (d. h. die Auslegung) gar nicht: nur in Verbindung mit der Himmelsreise findet sich dort die Brusteröffnung. Übrigens würde man; wenn sie wirklich ursprünglich nur eine buchstäbliche Erklärung von Sūrah 94, I wäre, erwarten müssen, daß man in den speziellen Brusteröffnungs-ladithen das Verbum ثرك

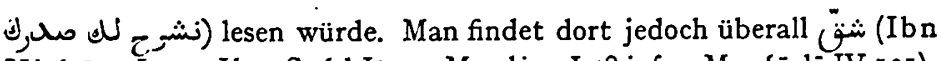
Hishām I 105, Ibn Sa'd Ix , 70, Muslim I 58 infra, Mas'ū dỉ IV I3I). 


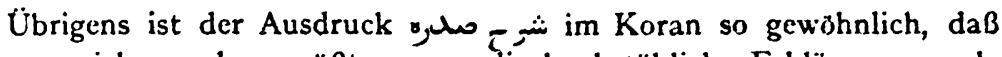
man sich wundern müßte, wenn die buchstäbliche Erklärung gerade auf 94, I zurückginge. Wir werden folglich untersuchen müssen: I. was die ursprüngliche Bedeutung der Himmelfahrts-Traditionen ist, 2. wie es mit der Verbindung Himmelfahrt-Brusteröffnung steht, 3. müssen wir spätere Zusätze ausscheiden und fragen: 4. wieweit hat sich die ursprüngliche Bedeutung erhalten?

\section{I.}
... customs which appear motiveless ... may receive quite a simple explanation from some circumstance still surviving amongst one or two primitive peoples. KenNe, Ethnology 219.

Betrachten wir zum Beispiel die Tradition, welche Tabari in seine Annalen (I II 57 ff.) aufgenommen hat. Dort wird erzählt, wie der Prophet einmal, als er sich seiner Gewohnheit gemäß bei der $\mathrm{Ka}$ 'bah schlafen gelegt hatte, von den Engeln Djibrīl und Mikā'il besucht wurde, die ihn auf den Rücken legten, ihm den Bauch spalteten, seine Eingeweide mit Zamzamwasser wuschen und von Polytheismus reinigten, ihn mit Glauben erfüllten und zum Himmel emporführten. Sie durchwanderten diesen, bis sie die Sidrah al Muntahä erreichten, wo dem Muhammed Offenbarungen von Allah zuteil wurden.

Dem Ethnologen sind derartige Erzählungen sehr wohl bekannt.

Von verschiedenen Volksstämmen Nord - und Zentral -Australiens berichten uns SPEncer und Gillen und vor ihnen z. B. Howitt über etwas Ähnliches bei der Initiation des Medizinmannes. Zwei von den zahlreichen Beispielen mögen genügen!

"In the Binbingatribe the doctors are supposed to be made by the spirits who are called Mundadji and Munkaninji, father and son. An old Thakomara man named Kurkutji who is a celebrated medicineman in the tribe told us that one day he walked unto a cave in a hill. ... The old Mundadji who unknown to him was wandering about came up, caught him by the neck and killed him. Mundadji. cut him open, right down the middle line, took out all of his insides and exchanged them for those of himself which he placed in the body of Kurkutji. At the same time he put a number of sacred stones in his body. After it was all over the younger spirit Munkaninji came up and restored him to life, told him that he was now a medicineman and showed him how to extract bones and other 
forms of evil magic out of men. Then he took him away up in to the sky"r).

Von dem Medizinmanne eines anderen Stammes sagen dieselben Forscher:

"When any man feels that he is capable of becoming ... (a medicineman) ... he ventures away from the camp ... until he comes to the mouth of the cave (in which the spirits live). Here, with considerable trepidation he lies down to sleep. ..." Die Geister töten ihn und - "the victim falls dead and is at once carried into the depths of the cave ... (in which) the (spirits) are supposed to live in perpetual sunshine and amongst streams of running water - a state of affairs which we may regard as the paradise of the Arunta native - . . Within the cave (the spirits remove) all the internal organs and provide the man with a completely new set. ... After which operation has been successfully performed he presently comes to life again $\|^{2}$ ).

Auch unter den Barée-sprechenden Toradja von ZentralCelebes finden wir eine parallele Erzählung bezüglich der Entstehung des Priesterinnenamtes. Die Glückliche, die später die erste Priesterin wurde, soll auf ihrem Krankenbette von zwei himmlischen Wesen besucht worden sein. Diese erlaubten nicht, daß. sie ihre alten Kleider mitnahm, sondern gaben ihr ein neues $\mathrm{Gew}$ and, Hut usw., wie die Priesterinnen sie heute noch tragen und trugen sie in die Luft empor, wo sie über ihre neue Wirksamkeit belehrt wurde3).

Auch bei einigen Kirgisenstämmen findet man über die Weihe des schamanistischen Baksa (Wunderarztes) in einer seiner Beschwörungen Ähnliches 4).

"Hab' in' ( = Haben inne) Himmel fünf der Geister,

Schneiden mich mit 40 Messern,

Stechen mich mit 40 Nadeln,

Und sie ließen mir am Scheitel

Einen langen Haarzopf wachsen!

Unterwarfen mich dem Dämon,

1) The Northern Tribes of Central Australia (1904) $487 \mathrm{ff}$.

2) Spencer and Gillen, Native Tribes of Central Australia (1899) 523 f.; weitere Belege für Australien findet man ebenda 522-530; ferner Northern Tribes 479-489; Howirt, JAnthr. I., XVI 47 ff.; vgl. BAstian, Allerlei I 252, 255 ff., 267. Mauss, L'origine des pouvoirs magiques dans les sociètes australiennes (1904).

3) KRUYT en AdriAni, I 375 (vgl, noch II 117).

4) RADLOFf, Das Schamanenthum und sein Kullus 65. 
Lehrten mich das Ungewohnte, Banden fest mich an den Kobus, Hießen mich den Segen sprechen.

Opferschaf mit gelben Köpfen

Ließen schlachten sie im Hause.

Fest im Körper setzten sie sich,

Drehten mir im Krampf die Glieder.

Auch die Zeremonien der muhammedanischen buginesischen Bissu's bei der Weihe eines neuen Adepten verdienen in dieser Hinsicht Beachtung. Jener muB eine Art Bad nehmen, durch das er längere Zeit auBer Bewu Btsein gerät. Während dieser Zeit schwebt er stets in höheren Sphären, um über das übernatürliche Wissen belehrt $z$ u werden $\mathrm{r}$ ).

Eine derartige Initialhimmelfahrt (bezw. Fahrt durch die Geisterwelt) kennen weiter - es möge hier genügen, nur noch ein paar Namen zu nennen, um auf die allgemeine Verbreitung dieses Phänomens hinzuweisen - die südsibirischen ${ }^{2}$ ) und amerikanischen Schamanen 3), die Medizinmänner von Zentral-Brasilien 4), die Priesterkandidaten der Niasser 5), die Batak ${ }^{6}$ ), die Zulus 7) und andere Völker Afrikas 8), kurz: viele Naturvölker (I), ja selbst die alten Inder 9), die Griechen in ihren Zauberpapyri ${ }^{\text {xo }}$ ) usw. "I)

Auch die ältere jüdische Literatur bietet einige sehr interessante Belege für unsere Himmelfahrt der Weihe. Denken wir an Jesaja VI, wo der Prophet von seiner Berufung berichtet. Er sicht den Herm auf einem hohen Throne sitzen. "Da flog zu mir einer von den Seraphim mit einem glühenden Stein in der Hand, den er mit einer Zange vom Altare genommen hatte"; damit berührte und reinigte dieser den Mund des Jesaja. "Da hörte ich die Stimme des Herrn, der da sprach:

r) Matthes, Over de Bissoe's 54. Für einen derartigen Initialritus mit Bad in Afrika vergleiche man Ellis, The Eure speaking peoples 143 f. (vgl. 106f., 109); The Tshi speaking peoples $131 \mathrm{ff}$.; The Yoruba speaking peoples 97 (vgl. $138 \mathrm{ff}$.).

2) JAnthr. I. XXIV 87 (vgl. 153).

3) Hodge, Handbook I 523 b; vgl. 522 a.

4) Von der Steinen, 343; vgl. 346.

5) TBGKW XXXIII 475 .

6) WARNeck, Religion der Batak 90.

7) Callaway, Zulutales $152 \mathrm{f}$., $316 \mathrm{ff}$.

8) Bartels, Medizin der Naturvölker 50, 78.

9) Z. B. Mahäbhärata III adhy. $168 \mathrm{ff}$; vgl. 42.

10) ARW IV 167.

11) Z. B. Tylor, Primitive Culture ' I 439; Bastian, Mensch II 319, 371; Année Sociologique VII $37 \mathrm{f}$. usw. 
Wen soll ich senden und wer soll für uns gehen? Ich sprach: Hier bin ich! Sende mich ! Ein anderes Beispiel ist die Weihe und Sendung des Propheten Hesekiel (Kap. I-3). Der Himmel hat sich ihm aufgetan und er schaut die Herrlichkeit Jahwes. Dieser entsendet ihn und spricht (2, $8 \mathrm{~b}$ ff.): Öffne deinen Mund und iß, was ich dir jetzt geben werde. $" \mathrm{Da}$ sah ich, wie eine Hand gegen mich ausgestreckt war, in der sich eine Buchrolle befand. ... Da offnete ich meinen Mund und er gab mir die Rolle zu essen (vgl. hierzu Frazer, Golden Bough 3, part V, vol. II, I43) und sprach zu mir: "Menschensohn, deinen Bauch sollst du sie verzehren lassen, und dein Innres sollst du mit dieser Rolle füllen, die ich dir übergebe «; und ich aß, und sie ward in meinem Munde so süß wie Honig.

Daraus ergeben sich uns folgende Schlüsse:

I. Tabari's Hadith enthält eine Initiationshimmelfahrt, was um so deutlicher ist, als er mit den Worten beginnt: تَّا كان

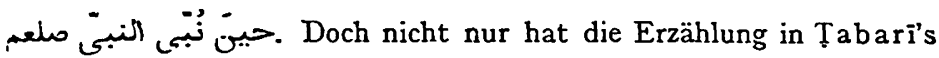
Annalen diesen Charakter, sondern dies ist auch die Bedeutung, welche die ältere Überlieferung ihr beilegt. Natürlich ist es an sich schon merknürdig, daß wir diesen mi rädj eigentlich gar nicht in die Muhammedbiographie chronologisch eingliedern können, wenn wir sie nicht mit der Nachtreise verbinden, die wir durchaus von ihr scheiden müssen $\mathrm{I}$ ), . und daß tatsächlich längere Zeit eine Richtung diese Auffassung aufrecht erhalten hat ${ }^{2}$ ), welche nach den zahlreichen ethnographischen Belegen wohl nicht erfunden sein kann. Wenn wir aber die bezüglichen Traditionen genau lesen, so bemerken wir, daß dem Propheten, als er die Sidrah erreicht hat, nicht nur Offenbarungen gegeben werden, sondern auch die Șalät wird vorgeschrieben. Übrigens wird in allen

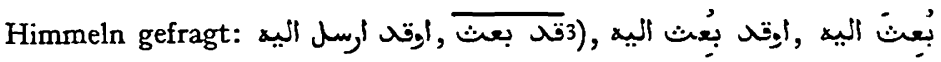
usw. Diese Frage 4) hätte selbstverständlich gar keine Bedeutung, wenn Muhammed schon lange des Prophetenamts waltete. Sonst würde man

》) NöLdere-Schwally, I 135 .

2) Selbst Baghawi ( $†$ 516), Leid. Handschr. Warner $394 \mathrm{c}$ Teil III Fol. $3^{\text {b }}$ kennt sie noch (vgl. die Anm. S. 29), wie auch die Sïrah al Halabijjah ed. Cairo 1292 I 488.

3) Z. B. Ibn Hish. 27i. Tah. Ann. I 1158. Tafs. XV 7, oben.

4) Aus NawawI, Sharh Muslin I 235 Z. 25 ff. scheint hervorzugehen, daB

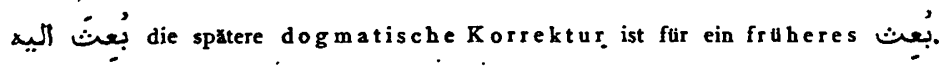
(Mitteilung ron Prof. Dr. C. Snouck Hurgonje, dessen Aufmerksamkeit von Prof. Bevan auf diese Stelle gelenkt wurde.) Jedenfalls bedeutet لئن usw. natürlich nur: ist Muhammed (von Allah) schon entboten? 
den Himmelsbewohnern wohl eine gar zu große Schläfrigkeit zumuten müssen, sehnen sie sich doch nach der Sendung des Muhammed. Auch findet. 'man in Tabari's Tafsir $(X V, 6)$ u. a. S. ausdrücklich behauptet, daß bei der Himmelsreise dem Propheten ختنم الننبوتة gegeben worden sei. Fügen wir hierzu außer der obigen Stelle aus den "Annalen * noch eine Tradition, die wir bei Buchārī, Muslim und in Tabari's Tafsir ${ }^{\mathrm{I}}$ ) finden, wo ebenfalls ausdrücklich gesagt wird: die Himmel-

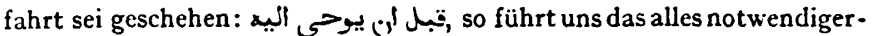
weise zu dem Schluß, daß die ursprüngliche Bedeutung des $m i^{c} r a \bar{d} j$ die Initiationshimmelfahrt ist.

II. Die ethnographischen Parallelen zeigen uns, daß die Brusteröffnungsgeschichte tatsächlich zu den Himmelfahrtserzählungen gehört. Wir müssen eigentlich drei Initiationsarten unterscheiden:

I. eine Körperreinigung,

2. eine Himmelfahrt,

3. eine Körperreinigung und Himmelfahrt.

Die erste Art der Weihe finden wir bei mehreren Völkern, z. B. bei einigen australischen Stämmen ${ }^{2}$ ).

Auch die Seedajaks in Borneo kennen sie nach Ling Rotr 3): A whole night's incantation is gone through . ... and in the morning the great function of initiation is carried out. The manangs lead the neophyte into a private apartment curtained off from public gaze by long pieces of native woven cloth; and there, as they assert, they cut his head open, take out his brains wash and restore them to give him a clear mind to penetrate into the mysteries of evil spirits and intricacies of disease; they insert gold dust into his eyes to give him keenness and strength of sight. ...."

Dergleichen teilt FRANCiscus COREAL in seinen Voyages aux Indes Occidentales nin Ansehung der Indianer de Rio de la Plata mit: "Wer unter ihnen ein Arzt oder Priester seyn wil, mus lange Zeit und ofermalen gefastet und unterschiedlichemale mit wilden Thieren insbesondre mit Tygern gekämpfet haben, und von ihnen gebissen oder wenigstens gerissen worden seyn. Nachmals kan er in den Priesterorden aufgenommen werden. Denn bey ihnen wird der Tyger vor heilig gehalten, und die Merkmale seiner Klauen gelten eben

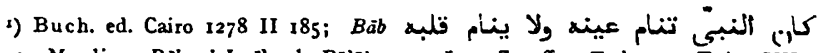
Trad. 2; Muslim, Bäb al-Isrä̉ ed. Būläk 1290 I 59 2. 3 ff.; Tabari, Tafsir XV 3 Z. $6 \mathrm{ff}$. infra.

3) SPENCER and GILLeN, Northern Tribes 484 .

3) LiNG Roth, Natives of Serawak I 281; vgl. $27 \mathrm{I}$. 
so viel, als bey uns ein Doctorhut. Hierauf wird ihm ein aus gewissen Kräutern distillirter Saft auf-die Augen getrippet; und dieses ist die priesterliche Salbung, nach welcher dieser neue Priester die Geister aller empfindlichen und materiellen Dinge beruhigen, mit ihnen geheime Gemeinschaft haben und sich ihrer Tugenden theilhaftig machen kan "I).

Eine solche Versetzung in den hellseherischen Zustand finden wir auch in Afrika 2).

Die zweite Art Initialritus ist, wie schon gesagt, die Himmelfahrt. Ihr geht immer eine Periode strengen Fastens voran. Auch bei der ersten Art der Weihe findet man diese. Dixon hat recht, wenn er über den Weg, die Würde eines Medizinmannes zu erwerben, folgendes sagt 3):

"It goes without saying almost, that the most wide spread method is that of fasting and solitude" (vgl. VIERKANDT, Natur und Kulturvolker, 278). "The weakening of the body and the stimulation of the imagination through these means is almost universally regarded as one of the most efficient ways of getting into communication with the unseen world. ... Very frequently there is added to this requirement of fasting and solitude that of bodily cleanliness. This may be attained by frequent bathing 4)... Not infrequently, also, this exterior cleanliness must be accompanied by an inner cleansing as well and we have then the use of various sorts of purgatives 5)."

Wir sehen also: der Gedanke ist, die körperliche Wohnung gründlieh zu reinigen, bevor und damit der neue Geist darin wohnen kann und will. Askese fördert die Entsagung des eigenen Willens und Unterwerfung unter die Geister (vgl. VIER KANDT, Natur und Kulturvölker, 279). Bisweilen findet man die äußerliche Reinigung nicht genügend, und wird die Erneuerung des Körpers betont, wie wir oben sahen.

Dies trifft nicht nur für Amerika, sondern auch für die primitiven .Völker aller Länder der Erde zu. Nirgends wird die Notwendigkeit der Reinigung durch Askese, bevor man die himmlischen Sphären

3) Nach BAUMgarten, Allgemeine Geschichte der Länder und Völker von Amerika (1752) I $\times 57$ (vgl. die Stelle aus den Lettres edifiantes ibid. $156 \mathrm{f}$ ) $=$ Lafiteau (holl. lusg. 1731), De Zeden der Wilden van Amerika I $164 \mathrm{f}$.

2) Elt1s, Yoruba speaking peoples $138 \mathrm{ff}$. Uber eine andere dauerhafte Initiation s. Frazer, Golden Bough II 30; vgl. die Bemerkung von SpEncer and Girien, Native Tribes 525; vgl. noch die Acta Sanctorum Bolland. z. B. über Sancta Theresa.

3) JJourn. Am. Folklore XXI 4:

1) Mahäbhärate III adhy. 168, Vrs. 21; KrUYT, Animisme 350; Ell.1s, Ewe speaking peoples 143; Lafiteau, op. cit. 131, 134, 168.

5) Lafiteau, op. cit. $165 \mathrm{f}$. 
betreten darf, schärfer betont, als in der indischen Literatur $\%$. In einer Stelle des Mahäbhärata ${ }^{2}$ ) heißt es: Dcm, der kein tapas (Askese) verrichtet hat, ist es nicht möglich, diesen großen gött. lichen Wagen (der ihn zum Himmel empor tragen wird) zu sehen oder auch zu berühren. geschweige denn zu besteigen, sagt Arjuna. Über diese Himmelfahrt als Initialritus ohne Wandlung des Körpers sind oben schon einige Beispiele mitgeteilt. So können wir uns zu der dritten Art der Weihe wenden. Bousset sagt in seiner trefflichen Abhandlung über die Himmelsreise der Seele 3), daß mit der Himmelfahrt verbunden ist die Vorstellung von himmlischen Gewändern oder Körpern, Salbung 4) usw., durch welche der Eintritt in diese überirdischen Regionen ermöglicht wird 5). Für das Stadium der Kultur, welches wir hier behandeln, gilt das nicht. Die Brusteröffnung selbst ist an sich schon, wie wir gesehen haben, eine Art Initialritus. Dasselbe gilt auch $z$. B. von den himmlischen Kleidern, die der ersten Barée-Toradja-Priesterin gegeben wurden, blieben diese doch seither die eigentümliche Tracht der Priesterinnen (oben S. 3). Hubert und Mauss ${ }^{6}$ ) haben denn auch recht, wenn sie nach einem eingehenden Studium dieser Vorstellungen bezüglich der Enthüllung magischer Kräfte durch die Geister unter verschiedenen Völkern, ihre Meinung, wie folgt, zusammenfassen:

"Nous voyons là l'image d'une révélation complète en plusieurs actes, comprenant une rénovation personelle, le transport dans le monde des esprits, l'acquisition de la science magique c. à. d. de la connaissance de l'univers. C'est au cours des dédoublements que s'acquièrent les pouvoirs magiques, mais ... ces dédoublements

1) Vgl. noch die interessante Geschichte im Rämāyana I sarg. 60 (ed. Bombay 1909, 99 f.).

2) Mahäbh. III adhy, 168 (ed. Bombay). nâtaptatapasā cakya eșa divyo makārathah draștum vâpy alhavā spraștum ärod̆hum kuta eva ca. Vgl. III $135: 16,167: 14$

3) ARIV IV 152; 2. Kor. I ff.

4) Daß die Salbung selbst noch als eine Weihe gefaßt wird, sieht man z. B. aus Weinel, Wirkungen des Geisles und der Geister 212; vgl. 197 und ARW IV 139, 235; s. übrigens 1. Reg. XIX 16; fesaja LX, I; vgl. Frazer, Golden Bough IV 66. Auch die himmlische Speise behält einen bleibenden EinfluB; vgl. Hesekiel II-III mit FrAzER Golden Bough part V vol. II 143 usw.

5) Auch die Askese ist nicht nur als Reinigung zu fassen, sondern auch als Surrogat der Weihe Nr. 1. Man vergleiche z. B. Rüm. I sarg. 6I-65 und viele andere Stellen (ucommon places a) der indischen Literatur (besonders lehrreich sind Stellen wie Räm. I 65 Vrs. 8 ff.). Lafiteau, op. cit. I 127, 172; Bastian, Mensch Ill 94; Achelis, Ekslase 15 ff., 113 f., I5 Iff.; Levvy BruHt, Fonctions Mentales $417 \mathrm{ff}$.

6) Année Sociologique VII [1902-1903] 38. 
initiatoires ne se produisent qu'une fois dans la vie du magicien qui en retire un bénéfice durable ${ }^{\mathrm{r}}$ ). "

Die Brusteröffnung ist in unserer Geschichte als eine Art irdischer Weihe zu verstehen, die in der Himmelfahrt ihre Ergänzung findet ${ }^{2}$ ). Wie sehr beide jedoch zusammenzuhalten sind, zeigen die zahlreichen Beispiele, welche die "rénovation personelle" ebenfalls nach dem Himmel verlegen 3).

LÉvy BRUHL 4) weist hin auf die große Ähnlichkeit zwischen den Zeremonien, welche die Weihung dieser Schamanen, Medizinmänner und Priester begleiten, mit den gewöhnlichen Pubertätsinitiationen und -träumen bei primitiven Völkern (Amerika, Afrika, Australien usw.) besitzen. Frazer 5) und Andere haben gezeigt, daß diese häufig nichts anderes sind, als eine Art Wiedergeburt.

I) Gegen Frazer, Magic art is I 101 .

2) $\mathrm{DaB}$ Brusteröffnung und Himmelfahrt zusammengehört haben, zeigt uns auch Ibn Sa‘d (I I, I.43), wenn wir diese Stelle mit Muslim I 58 ( = Nasāi ed. Cairo I3I2 I $78 \mathrm{f}$.) vergleichen. Bei Ib $\mathrm{S} \mathrm{Sa}^{\prime} \mathrm{d}$ lesen wir, daß die beiden Engel M. in seinem Hause fanden, ihn an den Ort zwischen makām Ibrahim und Zamzambrunnen führten und von dort mit einer Leiter, die wherbeigebracht war, zum Himmel emporstiegen. Diese Geschichte ist an sich schon verdächtig: warum soll man erst nach mak. Ibr. gehen, wenn doch

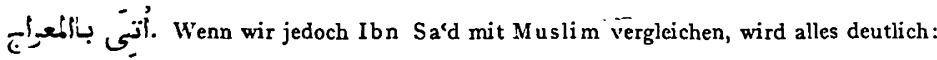

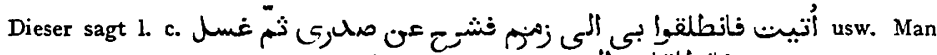

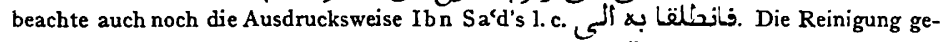
schieht ja mit Zamzamwasser. So wird in uns die Überzeugung reifen, daß Ibn $S a^{2} d$ 's Mitteilung nicht mehr ist als ein Exzerpt aus einer älteren Redaktion, in welcher die Brusteröffnung sich noch vorfand. Bei Ibn Sacd hat sich die Scheidung der beiden Stücke, über die wir.später (unten S. 27) sprechen werden, schon vollzogen. Auch Muslim kennt die Zugehörigkeit dieser Geschichte zum mi'rädj noch ganz gut. Die Stelle (I $58 \mathrm{f}$.), welche die Brusteröfinung bereits in die Jugendzeit verlegt, steht im $B a b$ al-Isra' (s. unten S. 13 ff.). - Übrigens findet man öfters bei den Initiationen ohne Luftreise schon den Anfang einer Himmelfahrt. Man berichtet, daß der Kandidat längere Zeit ohne Bewußtsein ist (z. B. in den schon oben angeführten Stellen aus Spencer and Gillen und Ellis, vgl. noch Lafiteau I i7 1 f. = Baumgarten, op. cit. I 163; Von der Steinen, op. cit. 343). Man weiß ja, daß den primitiven Völkern die Ohnmacht als ein Anzeichen dafür, daß die Seele sich entfernt hat (KRUYT, Animisme 75; Frazer, Golden Bough 3 II [191 I] 34 u. a. S.), odez auch als ein Zeichen von Besessenheit gilt (vgl. unten S. 23).

3) Oben S. 3, 4, 5; auch z. B. die Salbung bei Bousser, ARW IV 235.

4) LévY BRUHL, Les fonctions mentales dans les societés inférieures (1910) 417 . Es wurde leicht sein, auch für diese Pubertätsträume eine Fülle von Belegen beizubringen, die selbst den dikshä-Zeremonien (Oldenberg, Religion des Veda $40 \mathrm{r}$ ff.) der alten Inder zu entnehmen wären, sie sind jedoch wohl allbekannt, schon aus dem popularen Büchlein von Dr. Achelis: Ekstase.

5) The Belief in Immortalily (1913) I 254 f., $260 \mathrm{f.,} 290 \mathrm{f.,} 30 \mathrm{f} \mathrm{f.,} \mathrm{43I,} 434 \mathrm{f}$. 
nThe ceremony of initiation at puberty is very ofte regarded as a process of death and resurrection; the candidate is supposed to die or to be killed and to come to live again or be born again."

Wenn wir beachten, daß die primitiven Völker als Beweis oder "Quelle" ihrer Ideen über das Jenseits die Erzählungen der Scheintoten betrachten ${ }^{1}$ ), und man also erst durch den Tod gegangen sein muß, um das Jenseits zu kennen, daß viele Einweihungserzählungen genau diesen Charakter tragen ${ }^{2}$ ), ja wenn wir sehen, daß dieser Gedanke "of new birth", einer Erneuerung der Persönlichkeit, in diesem Falle, wo der Mensch von dem Augenblick an nicht mehr er selbst ist, sondern die Wohnung des Geistes (oder der Gottheit) wird, verschiedenen Völkern bekannt ist 3), wenn wir diese Tötung und Neubelebung bei unserer wichtigsten Parallele, der australischen 4), deren Ähnlichkeit mit unsrer Legende unverkennbar ist, ausdrücklich konstatiert finden, so müssen wir auch diese Erzählung hinsichtlich ihres Ursprunges 5) in gleicher Weise verstehen.

Der Zweck aller dieser Arten der Weihe ist nichts anderes, wie LÉvy BRUHL richtig sagt ${ }^{6}$ ), als "pour mettre ... (les candidats) en communion avec certains esprits 7) 4 .

1) Tylor, Primitive Cullure 3 II 46, 48; KRUYT, Animisme 359 wie auch das Erscheinen eines Verstorbenen im Traume, z. B. LiNG Roth, Natives of Serarcak I 232; KRU YT, op. cit. 72 ; Ellts, Yoruba speaking peoples 129 f.; FrAzER, Belief in Immortality I 27, $139 \mathrm{f.,} 213 \mathrm{f}$.

$\Rightarrow$ Bastian, Mensch III 135, 137 f. (Allerlei I I32); id. Allerlei I 256 (= Howitr, J.Inthr.I. XVI 1. c.), 274 f.; ders. Fava usw. 57; Achelis, Ekstase 56 f.; Lin. Roth, Natives of Serawak I 211 ff., 28I; Tylor, Primitive Cullure 3 II 50, 52; LÉvy Bruzl, Fonctions Mentales 417 ff.; MEINERS, Allgemeine kritische Geschichte der Religionen (1807) II 504; Lafiteau, op. cit. 142 ff.; Spencer and Gillen, 1. c.; Radloff, op. cit. 65; Matthes, Bissoe's 24; vgl. auch BTLV LXIX [1914] 369,379; Codrington, Melanesians 90, 93; Frobenius, Geheimbünde Afrikas (1894) in "Sammlung Gemeinverst. Wissensch. Vorträgee, Neue Folge, IX. Serie, Heft 209, II (vgl. ders., Masken und Geheimbünde. Abh. der K. Leop. Carol. Deutsch. Akad. d. Naturforschere [1898]); Schurtz, Allersklassen und Männerbünde (1902) 102-109, 354-361. Vgl. auch noch Dieterich Mithrasliturgies S. 157 ff., 233.

3) FrAzer, Magic Art I 380 (ganz merkwürdig); 1. S a muelis 10,9 (vgl. Eerdmans, "Theol: Tijdschr.4 1913, 120 f.), 2. Kor. 5, I ff.; Weiner, Wirkungen des Geistes und der Geister 92.

4) Oben S. 2.

5) D. h. im Heidentum. Vielleicht war die urspr. Bedeutung schon in M.s Zeit vergessen und kannte man $n$ ur noch die Sache selbst (vgl. unten S. 24f.).

6) Fonctions Mentales 42I; vgl. Frazer, Immortality I 432-434, vgl. noch Sürah 26: $193 f$.

7) Siehe unten S. 22 f.; Schurtz, op. cit. 102; vgl. I05; Codrington, Melunesians 71, 98 . 
II.

Doch kommt es auch vor, daß uns
jüngere Werke eine wunderlose Variante
darbieten von Berichten, die in älteren
Werken wunderhaltig sind.»
Horovitz in mIslam V, $44 \mathrm{f}$.

Wenn wir nun untersuchen, welche Züge in diesen $m i{ }^{i} r a \bar{d} j$ Traditionen entlehnt sind, so müssen wir erst die Brusteröffnung betrachten. Für diese ist gar kein Anhaltspunkt in der Literatur der Ahl al Kitäb zu finden ${ }^{\mathrm{r}}$. . Diese materielle Reinigung des Herzens und Erneuerung der Persönlichkeit deutet, wie wir sehen werden ${ }^{2}$ ), auf eine niedrigere Kulturstufe, als letztere Literaturgattung uns bietet.

Ausgangspunkt der Himmelfahrt.

I. Die mit der Nachtreise verknüpften Traditionen, die etwas polemisch gefärbt sind, nennen öfters das Haus der Umm Hâni’ ${ }^{3}$ ). Dies gehört jedoch zur Reise nach Jerusalem 4) (isrä).

2. Der Zyklus, der von Abū Dharr herstammen soll 5): es wird erzählt, wie das Dach von M.s Hause zu Mekka sich öffnete und Gabriel herniederstieg.

3. Die Hadîthe, die auf Mālik ibn Șa'șacah zurückgehen. Dort ${ }^{6}$ ) heißt es, daß er in Gesellschaft jemandes (oder mit den Ḳurai-

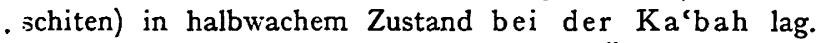

4. Eine Redaktion, die auf denselben Überlieferer zurückgeht 7); in ihr läßt man M. auf dem hiidjr (oder hatîm) liegen.

5. Eine sehr ausgeschmückte Tradition von Abü $\mathrm{Hu}$ rairah, ohne Ortsbestimmung, ganz sicher aus späterer Zeit ${ }^{8}$ ).

Es ist wahrscheinlich, $\mathrm{da} \beta$ in 49 ) eine Tendenz liegt. Bei der Erörterung des Verhältnisses 2wischen isrä und mi'räd $j$ werden ${ }^{10}$ )

r) BoussEt in seiner Studie über die Himmelsreise der Seele, die eine besonders reiche Materialsammlung enthält, ARW IV und Dieterich, Mithrasliturgie² 191 f.; vgl. Arda Viraf, ed. HAUG $15 \mathrm{I}$ - gegen BLochet RHR XL, x-25, 203-236 (vgl. Bousset, op. cit. $250 \mathrm{nt}$.).

2) Unten S. 22.

3) Tab. Tafs. XV 3 Trad. I (Jackūbì II 25).

4) Unten S. $14 \mathrm{f}$.

5) Muslim I 59; Buch. I 50 (Ibn Sa'd I I 143).

6) Buch. II 144; Musl. I 60; Tab. Tafs. XV 3 Trad. 2 (Ann. I I157). und 6.

7) Buch. II 221; Tab. Tafs. (mit abweichendem Lsnäd ausführlicher) XV 3 Trad. 5

8) Tab. Tats. XV 6 if.

9) Snoucr-Hurgronje, Mekka I 3, II.7o f. und Mekk. Feest $104 \mathrm{f}$.

ic) Unten S. 14. 
wir selbstverständlich 3 und die merkwürdige Redaktion behandeln, die sich ganz an den Sprachgebrauch von Sürah I7, I 1) anschlieBt, wo also von dem Masdjid al haräm gesprochen wird. Obwohl eine karaibische Parallele in vielem mit 2 übereinstimmt 2), müssen wir es vorläufig dahingestellt sein lassen, zu entscheiden, welche Redaktion die ursprüngliche Form der Erzählung enthält. Eine gleiche Verschiedenheit können wir bemerken hinsichtlich der Anzahl von Personen, die zu M. kommen. Bald wird Gabriel allein 3), bald er und Michael 4), bald wieder drei Personen genannt 5). In der Aus. gestaltung dieser Erzählungen werden fremde Muster nachgewirkt haben ${ }^{6}$.

Der (oder Die) Buräki ist kein wesentlicher Bestandteil der Legende; denn wo, wie öfters, $m i^{i} r a \bar{a} d j$ und $i s r \vec{a}$ verbunden werden, findet man zuweilen, daß die Reise von Jerusalem aus mit einer Leiter fortgesetzt wird 7 ), oder es wird in der reinen $m i^{i} r a \bar{a} j$-Erzählung die Leiter schon bei der Ka'bah aufgestellt ${ }^{8}$ ), oder Gabriel nimmt den M. bei der Hand 9). Buräk scheint in Vorstellung und vielleicht auch in Namen (!) auf Persisches zurückzugehen ${ }^{10}$ ).

Die Leiter geht wahrscheinlich indirekt"r) auf die bekannte Jakobsleiter zurück 12). Ursprünglich ist wohl keines von beiden. Vielleicht ist die Stelle, wo nur von einem schnellen Pferde gesprochen wird ${ }^{3}$ ), als ein Übergang zur Buräk-Vorstellung zu betrachten.

Beides ist wohl eine Materialisation, wie sie in den späteren Erzählungen so häufig ist ${ }^{4}$ ) und wie sie sonst in den Seelenreisen anderer Literaturen auch manchmal gefunden wird ${ }^{15}$ ). Ganz sicher ist mit

1) Buch. II I85; Musl. I 59; Tab. Tafs. XV 3 Trad. 7 .

2) Lafiteau, op. cit. I I69-17I = Baumgarten I 16r f.

3) Buch. II I44, 221 ; Musl. I 57 ff., auch anonym Tab. Tafs. XV 3 Trad. 2.

4) Tab. Ann. I I157; Ibn Sa'd I' I43.

5) Buch. II r85; Musl. I 59; Tab. Ta/s. XV 3.

6) BousSET, op. cit. 14I, 16I u. a. S.

7) Tab. Tats. XV 1o f.; Ibn Hish. I 268.

8) Ibn Sacd I'I+3.

9) B. 1 50; M. I 59 ; vgl. T. Ann. I 1157.

10) Blochet, RHR XL, 204 ff.; Goldzmer, RHR XLIII, 3.; Thorning, 28 nt. ( JACOB); die etymologischen Betrachtungen BLOCHET's op. cit. 212 ff. bedürfen der Berichtigung und sind nicht überzeugend.

13) Weines, op. cit. 176, 197. ZDMG LXVIII 8, 9.

12) A. Mürler, Der Islam im Morgen-und Abendland I 86. Vgl. jedoch noch Tab. Ta/s. XV 10.

33) Tab. Ta/s. XV 6.

14) A. Müller, op. cit. I $86 \mathrm{f}$. Vgl. jedoch Tab. Tafs. XV 10.

15) RAdLoff, Das Schamanenthum 22,36ff.; JAnthr. I. XLIV, 63, 72, 73; KRUYT, 
dem Auftreten Buräk̆'s in diesen Geschichten die für Muslime so interessante Frage verbunden, wie die Himmelsreise stattgefunden habe.

Die Frage ist ja, ob sie bidjasadihi ${ }^{\mathrm{x}}$ ) oder binafsihi wa-djismihi ${ }^{2}$ ) oder birülıihi und nicht bidjasadihi 3) geschehen sei 4).

$$
M i^{\text {‘'}} \bar{a} \bar{d} j \text { und Isrä'. }
$$

Weil an dieser Stelle öfters die Nachtreise in die micrädj-Erzählung eingeschaltet wird, scheint mir hier der richtige Augenblick gekommen, um über beide etwas zu bemerken, das bisher, so viel ich weiß, unbeachtet geblieben ist. Die Geschichte der Nachtreise nach Jerusalem (isrả) knüpft' bekanntlich an Sūrah I7, I an: سبكان آتذى أسرىى u.s. w. Warum soll aber المسبجد الاقصى الاقيى Jerusalem heißen? Diese Erklärung, für welche gar kein Grund besteht, scheint einer großen Reihe von Traditionen anzugehören, welche, in der Zeit 'Abd al Malik's erdichtet, "die Aufgabe haben, die besondere Würdigkeit des Heiligtums in Jerusalem, welche in der Umajjadenzeit [im Gegensatz zum Heiligen Gebiete, wo damals 'Abdallâh b. Zubejr waltete] in den Vordergrund gestellt wurde", zu erweisen 5).

Vielmehr haben wir in المّرة المنتهى (Sürah 53, 14; siehe unten S. 18, 20) einen allgemeinen unbestimmten und feierlichen Ausdruck zu sehen ${ }^{6}$ ). Wenn wir diese beachten, so ist

Animisme 85 f., 101 f., 107 f., 350, 494 ff.; Nieuwenhurs, Quer durch Borneo I 125 (vgl. KRUYt 88), vgl. 100 ebenda; Skent, Malay Magic 433; Wilken, Verspr. Geschr. III 238 bis 240; TyLor, Prim. Cult. II 51 usw. usw. Dieterich ${ }^{2}$ I83 $\mathrm{f}$.

1) Tab. Tafs. XV' 5. Vgl. BaghawI Tafsïr (Leid. Handschr.) III Fol. I. Z. 15 ff.

2) Ebend. 12.

3) Ebend. 12.

4) Burāk ist weiter das Reittier aller Propheten (passim) oder auch das Reittier Ibrāhim's speziell ( $\mathrm{T}$ ab. Tats. XV 5 Trad. I; vgl. WEIL, Biblische Legenden der Muselmänner 85); Muhammed wird es am Tage der Auferstehung wieder besteigen (WeIl 276; vgl. Wolfr, Eschat. 101 ff., arab. T. 57 f.). Auch Asiah soll es einmal geritten haben (ebend. 127). Die spätere Literatur kennt noch andere besondere Reittiere: Maimūn(ah) (WEIL 18, 27; WCLFF 12, a. T. 8) und Haizam (ebend. 153).

5) Goldziner, Muh. Siud. II $35 \mathrm{ff}$.

6) Es wäre an sich denkbar, daß in 17,1 mit بعبر irgend ein früherer Prophet (z. B. Henoch, vgl. 19, 57 f.) gemeint sei. Dies scheint mir jedoch nicht richtig, nicht nur, weil die gan ze Tradition diese Auffassung, soviel ich weiß, $n$ ich $t$ kennt und den Vers nu r entweder auf den miérä $j$ oder auf die Nachtreise nach- Jerusalem bezieht, sondern auch, weil im Koran mit عبرلنا oder, abgesehen von den Stellen, wo ausdrücklich ein anderer mit Namen genannt wird (19: $;$;8: 16, 40; $54: 9)$, nur auf Muhammed hingewiesen wird (2:21; 8: 42; 18: $1 ; 25: 1 ; 39: 37 ; 53: 10 ; 57: 9)$. Dies wird also wohl auch in $17: 1$ der Fall sein. 
es schon an sich deutlich, daß 17, I wahrscheinlich auf die Himmelfahrt anspielt. Auch im Himmel gibt es ja ein Heiligtum (unten S. I9 Anm. 5). Dies leuchtet um so mehr ein, als die ältere Tradition gar keinen Unterschied macht zwischen isrä und mírädj $\left.{ }^{1}\right)$. Betrachten wir z. B. Buchã ri im Bāb كيف فرضت الصلاة في الاسراء , wo (wie im $B \bar{a} b$ al mi'rādj, in dem über ليلة أسرى به gesprochen wird, ohne daß etwas 'über „ $i s r a{ }^{\prime}$ " ausgesagt wird) isrä ganz im Sinne von $m i^{`} r \bar{a} d j$ gebraucht wird 2 ). Am deutlichsten zeigt sich diese Auffassung noch in den $m i$ 'rädj-Traditionen, in welchen die Himmelfahrt von der $\mathrm{Ka}$ abah ihren Ausgang nimmt oder, wie es in einer Redaktion unzweideutig heißt: vom masdjid al harām (vgl. oben S. 12; für diese Ausdrucksweise vgl. Snouck Hurgronje, Mekkaansche Feest 39 Anm. und 44 Anm. 2). Wir sehen also, daß wir hier ursprünglich zwei Deutungen vor uns haben: 1. isrä' (dem Sprachgebrauche von I7, I entlehnt) $=m i^{i} r \bar{a} d j$, wo also gar keine Rede von einer Reise nach Jerusalem ist und I7, I demnach auf die Himmelfahrt bezogen wird (man vergleiche noch die verschiedenen Traditionen bei Ta b. Tafs. XV zur Stelle); 2. nachdem die umajjadische Auffassung rezipiert worden ist, unterscheidet man $i s r \bar{a}^{\prime}=$ Nachtreise nach Jerusalem, und $m^{i}{ }^{c} r \bar{a} j j=$ Himmelfahrt. Beide werden ofters miteinander verbunden.

Die Unterscheidung ist also nicht ursprünglich 3), sondern nur durch einen Grundsatz verursacht, der durchweg in der muhammedanischen Tradition zur Geltung kommt (SNouck-Hurgronje, Mahdi, 16):

"Es ist eine eigenthümliche Erscheinung, wie in den späteren muhammedanischen Traditionssammlungen fast über jede Frage mehrere einander widersprechende Überlieferungen mitgeteilt werden, welche nach dem Sinne des Sammlers alle maßgebend sein sollen. Die Sache verhält sich aber so: vor der Periode des Sammelns wurden über jeden fraglichen Punkt voneinander abweichende Aussprüche Muhammeds in Umlauf gesetzt; das war nun einmal die Form,

1) Also haben wir, abgeschen von Sūrab 53, über die wir später handeln, in $1 \%$, und 17, 95 einen koranischen Anhaltspunkt für die Himmelfahrt. Letztere Stelle hat erst wirklich Bedeutung, wenn sie auf etwas von Muhammed selbst Behauptetes anspielt, wie die vorangegangenen Verse.

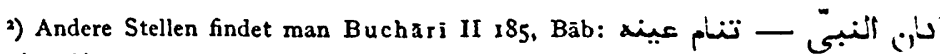
تلبن Trad. 2; Musl. I 59; Tab. Tafs. XV 3, XXVII 24; al-Nasā̄i I 78; Tirmidhi I 45; Mishkät 518, vgl. 521; Baghawi, Masăbilh II 177; vgl. noch Muslim. Bäb al-isrä'...ila 'l-samawät.

3) Auch die Überlieferung selbst unterscheidet sie öfters z. B. Tab. Tafs. XV5 Trad. I; vgl. NÖLDEKE-SCHWALLY I 135. 
in welcher jede Schule oder Partei ihre Ansichten kundgab. Die folgende Generation, des Kampfes über gewisse Gegenstände müde, nimmt dann in der Regel einen vermittelnden Standpunkt ein " [hier also leicht ermöglicht durch die zwei Wörter isrä' und mi'rädj, denen man einen verschiedenen Sinn beilegte] "und söhnt den Gegensatz aus durch Anerkennung der beiderseitigen Öberlieferungen, welche dazu aber harmonistisch, d. h. unhistorisch, interpretiert werden müssen. Genügt diese Operation nicht vollständig, so werden noch ein paar Traditionen, welche die Ansicht der Vermittler unzweideutig vertreten, hinzugemacht, und der Friede ist vollkommen."

Das war nun auch bei unseren Traditionen der Fall. Ursprünglich hatte man nur die richtige Erklärung, die I7, I auf die Himmelfahrt ( $m i^{\mathrm{c}} r a \bar{d} j$ ) bezog. Aus dem Sprachgebrauch entstand das synonyme isrā'. Die umajjadische Erklärung faßte I7, I als eine Verherrlichung des Heiligtums in Jerusalem. Der Idjmä erkannte beide Erzählungen an und fand die Möglichkeit dieser Anerkennung in der Differenzierung der beiden Wörter isrä und mi'rädj.

In der späteren Ausbildung der Muhammedbiographie nach dem Muster von und in der Konkurrenz mit der Biographie Jesu vertritt die Begegnung Muhammeds mit den Propheten in Jerusalem die Verklärung Jesu auf dem Thabor (Matth. I7, I ff., Mark. 9, I ff., Luk. 9, $28 \mathrm{ff}$.). Die polemische Absicht zeigt sich wie in den Mahditraditionen (SNouck-Hurgronje, Mahdi i7) in der Salät des Muhammed vor den Propheten. Man sieht, wie erst beide Er. $z$ ählungen parallel gelaufen sind: M.s Begegnung mit den Propheten und seine Überlegenheit über diese, welche sich zeigt hier in seiner Imāmwürde bei der Șalāt, dort in seinem Vorüberschreiten an Jesus und Moses (unten S. 16f.). So versteht man, wie mehrfach in den zusammengesetzten Legenden der Aufenthalt in Jerusalem seine eigentliche Bedeutung verliert (Bagh. II 178 infra) $x$ ).

Neben diesen beiden Auffassungen, von denen die numajjadische" die ältere ganz verdrängte, hat sich noch eine dritte, an 17,62 an. knüpfende, entwickelt, in $\left.\operatorname{der}^{2}\right)$ man $r u^{\prime} j \bar{a}$ mit isrä' zu erklären suchte.

Nun dreht man aber die Sache um.

Ist $r u^{\prime} j \vec{a}=i s r \vec{a}$, so ist $i s r \vec{a}$ eine $r u^{\prime} j \bar{a}$, aber wenn das der Fall ist und man $r u^{\prime} j a ̈$ als eine ekstatische Vision faßt, so ist es keine Reise mehr. So findet man häufig als Erklärung 3) von 17, I folgendes:

s) Anm. wahrend der Korrektur (s.. Nachschrift). Vgl. nunmehr BevaN in Wellhausen-Festschrift 56.

2) Vgl. Tab. Ta/s. XV 12.

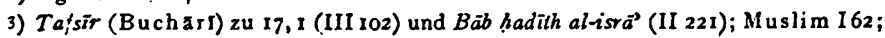
Tab. Ta/s. XV 5 Z. 14 v. u.; Mishkäl 522. 
nAls die Kuraischiten mir nicht glaubten, stand ich auf dem Hidjr. Da zeigte mir Allah Jerusalem und ich begann zu erzählen von seinen Ajät, während ich darauf schaute (so bekommt man die sru'jāin).

Haben wir oben gesehen, wie die Theologen die Differenz zwischen den beiden Auffassungen harmonisiert haben, so muB man die Schwierigkeit hier auf eine andere Weise lösen. Buchārī III 102 findet den Ausweg in einer Interpolation: حبي أسرى بى المى بيت المقلىس. Muslim I $62^{1}$ ) hat aber eine andere Möglichkeit erfunden: نقد رايتنى في في

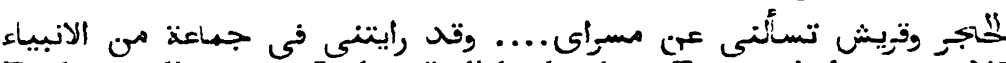
Er deutet die ganze Sache nämlich als einen Traum, indem er ru’jä in diesem Sinne faßt. Die Begegnung mit den Propheten, die man sonst nach Jerusalem verlegt, findet hier gleichfalls im Traume statt.

Das Wort فتنّ (17, 62) verursacht weiter die Erzählung von einem Abfall (dabei bewährt Abū Bakr sich selbstverständlich als al-Siddik), der sich sehr gut dem Unglauben der Kurais hiten angliedern läßt. Das öfters gebrauchte Verbum أïlerleichtert es uns, dem Ursprung dieser Erzählung nachzugehen. Bei Ibn Sacd (I I, I44) können wir noch sehen, wie diese Art Harmonisierung gewirkt hat: 1. Nachtreise, 2. Unglaube, 3. Muhammed will beweisen (hat aber die Einzelheiten vergessen!) und Allah zeigt ihm Jerusalem. Im arabischen Text ist die Naht der Zusammenflickung noch ganz gut wahrzunehmen (vgl. noch Tab. Tafs. XV 6f.).

Den hybridischen ${ }^{2}$ ) christlichen Himmelsreisen entlehnt sind wohl die bösen 3) (und kraft einer Tradition auch guten 4)) Mächte, denen M. auf seiner Reise begegnet, obgleich sie sich auch in anderen Religionen finden 5). Aber die Ähnlichkeit ist zu groß (Bousser, 1. c. I4I, 150, 154 usw.). Ist Sūrah 17, 46 u.a.S. zufolge die Anzahl von 7 Himmeln schon koranisch, die Beherrschung von je einem Gottes gesand ten ist ohne Zweifel die Muhammedanisierung älteren Musters ${ }^{6}$ ). Die Reihenfolge ist übrigens sehr vęrschieden. Dié gewöhnliche ist 7):

I Adam, II Jahịā und 'Îsā, III Jūsuf, IV Idrīs, V Hārūn, VI Mūsā, VII Ibrähīm.

1) = Mishkät 521 f.; Baghawi II 179. Viel ausführlicher und gewissermaßen abweichend ist schon al-Nasä'i I $77 \mathrm{f}$.

2) BousSET, ARW IV 150, 167, 232.

3) Z. B. Tab. Tafs. XV 10; vgl. Eph. 6, 12.

4) Tab. Tafs. XV 5 Trad. 2.

5) Nieuwenhuis, Quer durch Borneo I 104. KRUYt, Animisme 349 ff. u. a. S.

6) Bousset, 1. c. $237 \mathrm{ff}$., $268 \mathrm{ff}$. (vgl. ThORNing $268 \mathrm{ff}$.).

7) Buch. II I44, 221 f.; Musl. I 58,60; Mishkät 519; Baghawi II 177 f.; al-Nasä'ĩ I 76; Tab. Annales I 1158; Tab. Tats. XV 8. 
Daneben findet man die Verwechslung von II und III $x$ ) oder IV und $\mathrm{V}^{2}$ ) oder selbst VI und VII 3), welcher sich jedenfalls das Feilschen über die 50 Șalät's, mit dem jede richtige mi'rädj-Tradition schließt, besser angliedert. Kurz! Die Reihenfolge steht nicht fest, und die Traditionen haben wohl recht, welche dies ausdrücklich behaupten (Buch. I 50, Musl. I 59, Tab. Tafs. XV 4, Mishkät 521).

Nach dem, was Bousset ausgeführt hat, müßte man das Paradics in oder über dem VII. Himmel suchen. Dies findet man hie und da 4) wirklich. Eine andere Reihe von Redaktionen versetzt Paradies und Hölle in den I. Himmel 5). Wenn dies entlehnt wäre, müßten wir sie im III. Himmel antreffen ${ }^{6}$ ), aber da dies nicht der Fall ist, so läßt sich unter Beachtung des unmittelbar Vorangegangenen (die Beherrscher der sieben Himmel) schließen, daß in einer früheren Redaktion der mi'rädj-Traditionen die Reise durch die Himmel noch nicht so ausgebildet war, daß aber der Besuch von Paradies (und Hölle? ? 7)) zu der ursprünglichen Legende gehörte, wie es auch aus $\mathrm{Ibn} \mathrm{Sa}^{\mathrm{c} d}\left(\mathrm{I}^{\mathrm{I}}\right.$, I43) sich $\mathrm{zu}$ ergeben scheint (vgl. unten S. 20 über Sürah 53: 15).

In dem ersten Himmel sitzt Adam vor zwei Türen: wenn er die Leute von rechts durch ihre Pforte eintreten sieht, lacht er, aber über ${ }^{*}$ die Leute von links weint er ${ }^{8}$ ). Mehr in Übereinstimmung mit der Dogmatik ist eine andere Stelle 9), wo über die Geister gesprochen wird. So kann man auch die sonst ${ }^{\circ}$ ) ganz in der Luft hängende Perikope ${ }^{\text {II }}$ ) beibehalten, die das Schicksal der Wucherer, Unzuchtver-

3) Christlicher EinfluB? 2. Kor. 12, 2; BousSEt, 1. c. 234 ff.; Tab. Tafs. XV II; Baghawī II ${ }_{7} 8$ infra.. .

2) Tab. Tafs. XV 4 ; Musl. I 59 .

3) al-Nasā'ì I 78 .

4) Musl. I 59; Buch. I 50; Ibn Sa'd I ' 143; Ibn Hishām I 268; Tab. Ann. I I158; Tab. Tafs. XV 8, II. Bisweilen besucht Muhammed wie z. B. Mus I. I 59 f. das Paradies sowohl im ersten als auch im siebenten Himmel !!

5) Musl. I 59; Buch. I 50 f.; Tab. Tafs. XV 7 usw. Die Mālikfigur (vgl. Sūrah 43 : 77; z. B. Ibn Hish. I 268) wie Ismāīil (z. B. Tab: Tats. XV 10) und das Mädchen für Zaid im Paradiese (ebenda u. a. S.) werden wir als zu nebensächlich im Folgenden nicht zu besprechen brauchen.

6) BousSET, 1. c. $139,234 \mathrm{ff}$.

7) Nach dem, was Bousset l. c. 139 (vgl. 235) ausführt, scheint die Hölle in diesen Geschichten vielmehr aus christlichen Quellen eingeführt zu sein.

8) Buch. I 50, dem Sprachgebrauche von Sūrah 56, 26 ff. entlehnt; Mishkät 521; Baghawī II 179.

9) Ibn Hish. I 268; Tab. Ta/s. XV 10.

10) Tab. Tafs. XV 6.

27) Ebenda. 
brecher, Wegelagerer, Salät- und Zakät-Versäumer usw: usw. behandelt ').

Später 2) scheint es - in dogmatischem Sinne - am besten, gar nicht über das Paradies zu sprechen, weil es في اخخر الزمل, von Allah "herbeigeholt " werden wird (Wolff, Muh. Eschatologie ro5 Anm. 365).

Die Becher, welche dem M. auf dieser Reise meist3) in Jerusalem (in den $i s r a a^{\prime}-m i^{i} r a ̈ d j$-Traditionen), auch wohl im sicbenten Himmel 4 ) geboten werden, sind bald 5) virei, bald ${ }^{6}$ ) zu ei an Zahl. Für beide Mitteilungen findet man in der christlichen $u$. a. Literatur die Parallelen, deren Vorbild hier eingewirkt hat (vgl. Bousser). Jene scheint besonders, wahrscheinlich indirekt, von der Arda Viraf-Geschichte beeinflußt zu sein (op. cit. 15I). Für Arda Viraf sind die Becher noch das narkotische Mittel, um seine Visionen zu erhalten, wie man auch sonst 7) allerlei für diesen Zweck gebraucht. Bei Muhammed aber nicht. Das Anerbieten der drei (zwei) Becher ist für ihn eine Prüfung: er trinkt nur den mit Milch gefüllten $\left.{ }^{~}\right)$ und läßt die beiden anderen, welche Wasser ?) und Wein enthalten, unbeachtet. Man verbindet diese Geschichte wohl mit dem Weinverbot ${ }^{10}$ ): in einer Überlieferung trinkt $M$. darum auch den Becher mit Wasser ${ }^{\mathrm{Ix}}$ ). Weil er sich satt getrunken hat, nicht weil er, wie es sonst heißt 12 ), zur richtigen Wahl geleitet worden ist, lehnt er an dieser Stelle den dritten ab.

Die Sidrah al Miuntalia, die entweder oberhalb des VII. Himmels ist (z. B. Nasā'i I 76) oder in den VII. Himmel - so am häufigsten -

2) Das Thema scheint wohl aus christlichen Quellen herzustammen. Bousser, op. cit. 161 f. (vgl. jedoch Arda Virat 170 ff.).

2) Buch. II I44, 222; Musl. I 58,60 (Ja kūbī II 25 ist exzerptartig); Tab. Tafs. $\mathrm{XV} 4$.

3) Musl. I 57; Ibn Hish. I 263 f.; Tab. Tafs. XV 5, 7, 10, 12 (Buch. III 102; Musl. I 6r; Tab. Ta/s. XV 5).

4) Buch. II 222; Musl. I 60.

5) Buch. II 222; Ibn Hish. I 263; Tab. Tafs. XV 5, 7; Baghawi II 178 infra.

6) Buch. III 102; Musl. I 57,60f.; Ibn Hish. I 264; Tab. Tafs. XV 5, 10, 12; Baghawi II 178 .

7) Vgl. Achels, Ekstase 6 fi.; Bousset, op. cit. 163; TrLor, Prim. Cult. ${ }^{2}$ I 446, II 410 ; JAnthr. I. XXIV 93, XXVIII 258; Müller, Amerik. Urreligionen (2. Ausg.) 182, 217, 279 f., 656; Lafiteau, op. cit. I64; Meiners, Kritische Geschichte der Religionen II 493 f.; Frazer, Golden Bough: Magic Art I $318 \mathrm{ff} ., 381 \mathrm{ff}$.

8) Tab. Tats. XV 5 u. a. S.

9) Für Buch. II 222 s. unten S. I9.

10) Tab. Tafs. XV 7; Ibn Hish. I 264.

i) Tab. Tafs. XV 7 .

23) XV 10, 12 (Trad. 4). Auch bei Tirmidhi (ed. Kairo 1292) II 46 finden wir in einem Traume noch eine Reminiszenz an die Bechergeschichte. 
oder auch in den VI. I) versetzt wird, gehört zu Sūrah 53, I4ff., wie alles, was über sie mitgeteilt wird, eine Ausschmückung dieser Koranverse ist ${ }^{2}$ ).

Anderen Mustern entlehnt scheinen mir die Ströme zu sein, welche sich in der Nähe des Paradiesbaumes befinden (I. Mose 2, Io ff.; Apok. 22, 2). Die Nachrichten über diesen Gegenstand sind jedoch wieder verschieden.

I. Meist werden vier genannt, von denen $z$ wei, bälināni, im Paradiese selbst fließen, und die zwei anderen, $z \bar{a} h i r a \bar{n} i,=$ Nil und Eufrat sind 3 ).

2. In einer anderen Tradition liegen die 'unsur der letzteren im II. Himmel (Tab. Tafs. XV 4).

3. An einer anderen Stelle4) verwandeln sie sich nach Sürah 47 : $16 \mathrm{ff}$. in Ströme von Wasser, Wein, Milch und Honig (Wolff $192 \mathrm{Anm}$. 383; 193-195; arab. T. 106ff. DIETERICH ${ }^{2}$ I99); eine Redaktion der Bechergeschichte schließt. sich dabei an und spricht auch von einem Becher mit Honig (Buch. II 222, Mishkät 519, Bagh. II I78f).

4.: Auch werden wohl drei Ströme genannt, in welchen die Gläubigen sich waschen: Ra⿳⺈mah, Ni'mah und " Sūrah 76:21 (Tab. Tafs. XV 8).

5. Auf einer anderen Stelle heißen sie-Nahr al-Ralimah und Kauthar (ebend. II); dem Koran I08: I entlehnt (vgl. Baiḍāwĩ zu dieser . Stelle; WoLfF I co ff., a. T. I05 ff.).

6. Kauthar liegt im III. Himmel (ebend. 4). Vgl.WolfF I 9 I, Anm. 380. Nun 5) erhält M. seine Offenbarungen. Die Traditionen erwähnen gewöhnlich die Verpflichtung der fünf Șaläts ${ }^{6}$ ) und äußern sich in den suggestiven, aber dunklen Worten von Sürah 53, 10.

Eine Überlieferung 7) nennt außerdem noch die letzten Verse

) Musl. I 62; Nasā̄ì I 78; Mishkät 521; Bagh. II 179.

2) Den wundervollen Duft, welchen dieser Baum im Christentum (ZDMG LXVIII ro ff., Weinel, Wirkungen $198 \mathrm{nt}$.) besitzt, hat er auch im Islam beibehalten! Vgl. noch WolfF 23 Anm. 32.

3) Buch. II I44, 222; Musl. I 60; Tab. Tafs. XXVII 30; Mishk. 5I9; Bagh. II 178.

4) Tab. Tafs. XV 8, XXVII 29.

5) Erst besucht jedoch Muhammed den Bait al-ma'mūr (z. B. Baghawī II 178, Mishkät 519; vgl. Testam. Levi, ed. Sinker 140; Wolfr 5 Ánm. 8; G hazālī Durrah (ed. GAutier) 30 Anm. I), wo er, wie man öfters mitgeteilt findet, den Abraham trifft (Bagh. II 1;8 infra, Mishkät 520).

6) Ausführlicher bei al-Nasā'ĩ I 78. Für die Bedeutung dieser Geschichte, in der das Feilschen Muhammeds mit Allah auf Anregung Moses über die Anzahl der Șalawät mitgeteilt wird, vgl. GordzлнER, Muh. Stud. I 36. Die Dialoge zwischen Allah und Muhammed werden später stark ausgestaltet. SNoucr-Hurgron JE, Mekka II 7 I, JUYNBoll, Handbuch 131; vgl. Leidener Handschrift Warner 786 (7).

7) Musl. I 62; al-Nasã̄i I 78 Trad. 2; Mishkät 521 . 
von Sūrah II und die Zusicherung der Vergebung an alle Mluslimen, die sich vom shirk ferngehalten haben. Allzu große Neugier befriedigt eine andere, indem sie Allah eine salbungsvolle Predigt abhalten läßt ").

Da die Hadîthe an diesem Punkte gern Stellen wie Sūrah 53, 8f. 3) anführen, so müssen wir bemerken, daß hier die für den Mluslim so wichtige Frage gestellt wird, ob M. Allah gesehen habe oder nicht 3). Es entwickelt sich ja die Neigung, die Verse auf Gabriel zu beziehen. Bezieht man sie auf Allah, so bleibt noch die Frage übrig, ob er ihn mit seinem inneren Auge 4) oder mit dem leiblichen geschaut habe. Gegen die letztere Auffassung führt selbst die Überlieferung 5) Sūrah 6,203 und 42, 50 an.

Vielleicht (indirekt?) an altpersische Vorstellungen ${ }^{6}$ ) anknüpfend, lösen andere die Frage durch die Behauptung, M. habe Allah mit einem hidjäb von Licht gesehen 7). Aus der Gabriel-Erklärung entsteht nachher die Vorstellung: M. habe ihn bei dieser Gelegenheit in seiner majestätischen Engelrüstung gesehen ${ }^{8}$ ). Sehen wir jedoch, was der Koran zur Stelle selber sagt: Allah (Muhammed) bezeugt hier feierlich in Sürah 53, daß eine majestätische Gestalt ihn zweimal über den Koran belehrt habe. Das eine Mal sah er ihn بلافت الاعلى (53, 7) oder بالافن المبين (81, 23); das andere Mal bei der Sidrah al-Muntahā عis. Wenn wir nun untersuchen wollen, wer das erhabene Wesen sein solle, von dem M. spricht, so müssen

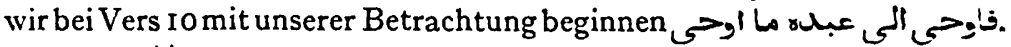
Aus dem Worte عبد geht am deutlichsten hervor, daß Allah Subjekt sein muß (vgl. 57:9): Muhammed glaubte also ursprünglich selbst, Allah gesehen zu haben (vgl. 40, I; $87: 6$ ). Aus Vers 13 ff. ersehen wir, daß M. tatsächlich behauptet, im Paradiese gewesen zu sein - nicht weil die Sidrah's dem koranischen Sprachgebrauche nach sich nur in göttlichen Gärten finden $(53,14,16 ; 56,27 ; 34,15)$, sondern weil das Wort $m a^{2} w a ̈ a$ (vgl. noch Wolff 190, ar. T. 105) im Koran an eiller Fülle von Stellen nur von Hölle $(3,144,156,196 ; 4,99,120 ; 5,76 ; 8,16 ; 9,74,96$; Io, 8 ; 13,$18 ; 17,99 ; 24,56 ; 29,24 ; 32,20 ; 45,33 ; 57,14 ; 66,9 ; 79,39)$ und Paradies $(32,19 ; 53,15 ; 79,41)$ gebraucht wird.

3) Tab. Tats. XV $8 \mathrm{f}$.

2) Tab. Ann. I $1158 \mathrm{f}$.

3) Für das Folgende vgl. Tab. Tafs. XXVII zu Sūrah 53. $53,13)$.

4) Buch. III 132; Musl. I 63; Tab. Tafs. XXVII 25 (infra)f. (zu 53, 11), 28 (zu

5) Tab. Tafs. XXVII zu 53, 12; Musl. I 63.

6) Arda Viraf 203.

7) Musl. I 64.

8) Z. B. Tab. Tafs. XXVII 31, vgl. 25. 
Unsere bisherige Untersuchung führt uns also $z u$ dem Schluß, daß Brusteröffnung und Himmelfahrt untrennbar zusammengehören (oben S. $6 \mathrm{ff}$.), daß die Geschichte der Himmelsreise in früherer Zeit weniger ausgebildet war (oben S. I7), drei Anhaltspunkte im Koran hat (oben S. I4 Anm. I) und mit einem Besuch im Paradiese von Anfarig an verbunden gewesen ist, und daß wir in der ganzen Erzählung eine Initiation in die prophetischen Funktionen zu sehen haben, wie z. B. fesaja VI.

Geht also die Erzählung auf Mohammed zurück, so müssen wir noch nach einer richtigen Bemerkung WeINEL's ${ }^{x}$ - weil stets "bei Vi sionen die objektiven Elemente, aus denen sie sich $z$ u. sammensetzen, der Gedanken- und Vorstellungswelt des Visionärs, die ihm von außen gegeben ist, entnommen sind - danach fragen, aus welcher Gedankenwelt in M.s Umgebung diese Vorstellungen stammen ${ }^{2}$ ).

Weiter werden wir noch versuchen müssen zu erklären, wie es möglich ist, daß man wohl für die Himmelfahrt, nicht aber für die Brusteröffnung die Belege im Koran findet, obwohl beide, wie wir oben ausführlich dargelegt haben, nicht $z u$ scheiden sind.

Zum Schluß müssen wir untersuchen, auf welche Weise die Verbindung $z$ wischen den beiden Teilen der Geschichte sich endlich gelöst hat, und die spätere Schicksale beider zeichnen.

III.

Diesem Kreise der alten arabischen Wahrsager stand Muhammed anfangs näher als man gemeinhin glaubt."

BEcker, Christentum und Islam Io.

Für die Entlehnung dieser Vorstellungen gibt es zwei Möglichkeiten: entweder sind sie aus dem judäo-christlichen oder aus dem altarabischen Kulturkreise herzuleiten.

Betrachtet man die unter den Himmelfahrten der "Schriftbesitzer ", die für unseren $Z$ weck in Betracht kommen, namentlich die Initialhimmelsreise, so findet sich, daß diese eine ganz andere Gestalt haben. Wir müssen von vórnherein beachten, daß nur diejenigen in Betracht zu ziehen sind, in welchen von einer Person gesprochen wird, die durch die Himmelfahrt zu einer neuen Funktion berufen wird, wie dies in der ältesten Fassung des Mi'rädj oder in Fesaja VI und Hesekiel I-III geschieht. Etwas ganz anderes bietet schon das Buch Henoch

3) Wirkungen des Geistes usw. 197 supra; Troo, Prim. Cult. 2 I 306.

2) Natürlich haben wir erst auch noch zu prüfen, ob das Ganze nicht eine Ausschmückung der koranischen Stoffe ist, wie wir die Tendenz dazu in späterer Zeit bemerkten. 
oder eine etwaige Ausschmückung von 11. Kor. 12, 2 ff. Dasselbe kann man von der Baruchapokalypse ") oder dem 25. Kapitel der Vila Adae et Evae sagen 2). Auch hier finden wir cine Art Himmelfahrt, die insofern eine Parallele zu den späteren mi'rädj-Legenden bietet, aber das, was wir als den ursprünglichen Charakter der micrädjErzählung erkannten: die Einweihung in die zukünftige Prophetenlaufbahn 3), finden wir dort gar nicht. Von der ganzen apokryphen Literatur kommt also nur eine Stelle aus dem Testamentum Levi f) in Betracht. Dort wird erzählt, wie Levi im Schlafe durch die Himmel fährt und dort Offenbarungen erhält, indem er zum Priester des Herrn gesalbt (Kap. 8, Ausg. 142) und beauftragt wird, den Menschen seine pustrpta zu verkünden (Kap. 2. Ausg. 139). Wie groß ist aber der Unterschied zwischen dieser Erzählung und unserer mírädj. Geschichte mit ihrer materiellen Erneuerung der ganzen Person $\mathrm{Mu}$ hammeds! Am nächsten stehen dem ursprünglichen Charakter der mi'rādj-Traditionen die Berufungen Jesajas und Hesekiels; aber auch diese bieten, wie man sofort sehen kann, nur eine Parallele zu der mi'rädj-Geschichte, nicht ihre Quelle (vgl. unten S. 2I).

Wenden wir uns nun dem zweiten Gedankenkreise Muhammeds zu.

Weil wir leider wenig Zuverlässiges über die religiöse Vorstellungs welt der vorislamischen Araber wissen, muß die Ethnologie die Angaben aufhellen: Wie z. B. der malaische Pawang seinen hantu pusaka

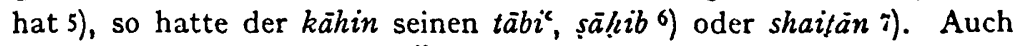
Gabriel wird wohl in der Überlieferung ${ }^{8}$ ) der Shaitän Muhammeds

1) Texts and Studies ed. Robinson vol. V no. I; Apocrypha Ancedota II by JAmes 84 ff.; wachr. d. Königl. Gesellsch. d. Wissensch. zu Göttingen 1896, 94 f́. - Prof. Dr. A. J. Wensinck hatte die außerordentliche Freundlichkeit, meine Aufmerksamkeit auf diese und diz folgenden Stellen zu lenken, wofür ich ihm hier meinen aufrichtigsten Dank sage. Vgl. auch BJusset ARW IV und Dieterick 2 igi ff.

2) ed. MeYer, Abh. Phil.-Hist. Klasse. d. Königl. Bayerischen Akad. d. Wissensch. Bd. XIV 229 (Kap. 25), fehlt im griech. Text. Apocal. Apocrypil. ed. Tischendokf iff. (Apoc. Mosis).

3) Vgl. oben S. 5 und unten S. 24; vgl. auch oben S. 8 die Ausführungen Bousset's über die himmlischen Kleider usw. in seinen Geschichten sowie meine Bemerkungen dazu.

4) The Testaments of the XII. Patriarchs ed. Sinker 138 ff. Vgl. auch noch Od. Salom. XI und Dieterich ${ }^{2} 179 \mathrm{ff}$.

5) SкEAt, Malay Magic 59; für Sibirien vgl. JAnthr. I. XXIV 65 (vgl. 64), 75. 85 f., 95, 129 u. a. S. Sieroszewskit, RHR XLVI (1902) 312 ff.; für Amerika Müllek, Urreligionen 217; "Journ. Am. Folkl. VI 39; ROchefort 418, 421 ; für Zentralafrika JAnthr. I. XXXII: 91 ; XXXVII: 159 f., 162 . Vgl noch FRA2ER Immortality I 308f., 322, 3 SS ff.

6) Wellhausen, Reste 234 Anm. 2; vgl. Goldziner, Abhandlungen I 5 .

7) VAN Vloten, WZKM XVII I8j f.; vgl. noch SPRenger, Leben I, 300. Sürah $26: 210,221 \mathrm{ff}$.

8) Wellanugen, 1. C. 
genannt, was sich folglich dieser Vorstellungsreihe anschließt (vgl. Sūrah 26: I93f.). Die Kähine redeten in sadj und nannten sich selbst "du", wenn sie im Namen des Geistes sprachen I). Wenn sie als Seher wahrsagten, so verhüllten sie sich. Sie wundern sich über das, was sie selbst sagen ( $m \bar{a} a d r a \bar{k} k a)^{2}$ ). Gern bekräftigen sie ihre Wörter mit Eiden bei der Sonne, dem Mond, den Sternen, beim Abend und Morgen usw. 3).

Wir wissen, daß dies alles auch sowohl von Muhammed als von vielen primitiven Medizinmännern bekannt ist. Auch sind bei solchen Leuten epileptische Anfälle sehr häufig 4): das in-Ohnmacht-fallen gilt ja den Naturvölkern als ein Anzeichen, daß die Seele sich entfernt hat 5) oder daß ein Geist in den Betreffenden gefahren ist $\left.{ }^{6}\right)$. Darum wird bei der Initiation öfters der Bewußtlosigkeit des Kandidaten Erwähnung getan 7). Hieraus hat sich nun auch die Theorie des Schamanisierens weiter gebildet: der Schamane schickt entweder seine eigene Seele oder die des "Guardian spirit " aus ${ }^{8}$ ), die ihm über die Frage Auskunft geben. Das letztere ist auch mit den Kähinen der Fall gewesen \%).

Wenn der primitive Mensch sich mit den Geistern in Verbindung setzen will, um von ihnen eine Offenbarung zu erhalten, so begibt er sich auf die Berge ${ }^{10}$ ), zu einem Grab ${ }^{\text {II }}$ ) oder einer heiligen Stätte ${ }^{12}$ ) usw., um dort Askese zu treiben ${ }^{13}$ ). Auch dies hören wir von den Kähinen

5) Vgl. Weinel, Wirkungen usw. 92. Lammens, Berceau 370 zu 257 und JAnthr. I. XXIV : 78,94 .

2) Vgl. Mikhailovskir, JAnthr.I 1895 (XXIV) 73, 146 .

3) Wellhausen, Reste 134 f.

4) Bastian, Allerlei I i3I f.; Radloff, 17, 50, 55; JAnthr. I. XXIV 65, I52 usw.; MEINERs, Allgemeine kritische Geschichte der Religionen (1807) II 488; Elliss, Polynesian Researches I 373 usw.; .vgl. WeINEL $129 \mathrm{ff}$. FRAzer Immortality I 196, 300, $368 \mathrm{ff} .388$.

5) KRUYT, Animisme 75; ElL1s, Ewe speaking peoples 106 f.; FRAZER, Immortality I 315; JAnthr. I XXXII: 91.

6) Lafiteau 170; Frazer, Immortality I 442; Magic Art I 377 f. Lammens Bercean de l'Islam $204 \mathrm{f}$.

7) Oben S. 9 Anm. 2.

8) Journ. Am. Folklore XXI 6, 39, beides schon bei den eigentlichen Schamanen in Sibirien: Miкна1Lovskir, JAnthr. I. 1895 (XXIV), 73, 143 und passim in dieser ausgezeichneten Abhandlung über Schamanismus; vgl. auch RHR XLVI 310.

9) Sūrah 6, 112, 121; 34, 13.

20) KrUYt, Animisme 72 f., 450, 517 ; Adriani en KrUYt I 255; Ling Roth I 287 .

21) Skent, Malay Magic 7I; "Zeitschr. f. Ethnol.* XXI 145 Anm. 27.

12) Wilken, Verspr. Geschr. III 334 ff., I 90 (Anm. 28).

13) Achelis, Ekstase 15, 16, 113 f., 151 ff.; TyLor, Prim. Cult. 3 II 413; Skent, Malay Magic 71; AdrinNI und KrUYt, I 255; KrUYt, Animisme 72f., 517; Ellis, Ewe speaking peoples 150; "Journ. Am. Folklore XVIII, 271; XXI, 4; MEINERS, II 141-150 489; 1. Mose 32, 24-32, vgl. Hosea 11, 4 f.; Oldenberg, Veda 404-407; Mahäbhärata 
und Muhammed berichten $\left.{ }^{3}\right)$. Obschon şaum nicht genuinarabisch ist, besagt die Entlehnung eines Wortes nicht immer etwas über die des $\mathrm{Be}$ griffes 2 ). Die Ethnologie bestreitet uns das Recht, die Geschichtlichkeit dieser Angabe anzuzweifeln. Außerdem ist es nicht unwahrscheinlich, daß

hinter dem تخحنَت der Mekkaner, so gefaßt, etwas Historisches steckt 3), da sie doch auch das itikäf kannten 4).

Die Folgen solcher asketischen Bestrebungen sind natürlich Träume und Visionen5), Charakter haben 7). In Sürah 53 spricht Muhammed über die Quelle, aus der er seine Wissenschaft gesammelt habe. Wie solche Traumerscheinungen auch den Kähinen und $S h \bar{a}^{-} i r^{\prime} s^{8}$ ) als Initiierungsart

III adhy 135 Vrs. 16 b, 20 b; adhy 167 Vrs. I4 (ed. Bombay); TBGKW L, 211 ; XLVIII 536, 545; Ling Roth I 287; Bastian, Allerlei I 132; Codrington, Melanesians 8r, 86, 93 ff.; "Journ. Am. Folklore XXI 4, vgl. noch oben S. 7 f.; Müller, Amerik. Urrelig. 79. 181, 183, 214, 650 ff.; Meiners, II 143, 161, 515; Baumgarten, I 85, 155 ff., 161; Frazer, Golden Bough 3 VI (1913) 377; Brinton, American Hero mylhs 129; Rochefort 424 f.; Acta Apost. 13, 2 (vgl. Weine L, 101).

3) Van Vloten, WZKM VII 236; vgl. Oldenaerg, Veda $405 \mathrm{f}$.

2) Wilken, Verspr. Geschr. IV 646 Index s. v. ontleeninge. Zu den dort verzeichneten Stellen ist I 88 (Anm. 23) zuzufügen; man vergleiche den Gebrauch des Sanskritwortes tapas (Askese) in verschiedenen malayo-polynesischen Sprachen für einen Begriff, den sie schon längst kannten.

3) Tab. Annales I i 47 u. a. S. Vgl. noch Houtsss, Versl. Mleded. K. Akad. Wetensch. Afd. Lett. (Amsterdam) IV Rks. II II, 24, 26.

4) Vgl. JuYnboll, Handbuch des islamischen Geselzes 125 Anm.

5) Acheris, Ekstase I. Kapitel; "Journ. Am. Folkl. \&XXI, 3; JAnthr.I. XIX, 293; LinG Roth I 231; Lafiteau 165; Rand, Micmactales 425. Vgl. Sürah 17:81 vgl. 19:57, 5 S. Die Geister wählen den Kandidaten. Wibken, III 364; Frazer, Immortality I 32.2, Magic Art I 378; Ellis, Eree speaking peoples 1. c.; JAnthr.I. XXXVII, 165, vgl. die Tradition, clie Caetani, Annali I $229 \mathrm{f}$. anführt und was über M.s Rival gesagt wird bei Sprenger, Leben Muhammeds I $117 \mathrm{ff}$. Die Weihe durch die Geister ist, wie wir schon bemerkt, sehr häufig. Vgl. noch: BoAs in $\bowtie$ Rep. Brit. Assoc. Adv. Sci.» r889 @ Journ. Am. Folkl.»XIV 95); Codrington, Melanesians 76; JAnthr.I. XXXII, 91; Elliss, Polynesian Researches IV 335 f. [Vgl. jetzt auch Hartmann, Türk. Bibl. Bd. XVIII I+5.]

6) 2. B. Musl. I. 56 f. [Vgl. nunmehr die Bemerkung R. Hartmanns op. cit. 157 Z. 15 It.]

7) Nöldeke-Schwally, Geschichte des Qor'äns 78, 70, 82.

8) GoldziHER, Abhandlungen I I8, macht wohl richtig einen Unterschied zwischen den Funktionen des $K a ̈ h i n$ und des $S h a^{-} i r$. Jedoch sind sie beide nach dem, was wir oben dargestellt haben, als Schamanen oder Medizinmänner zu fassen, weil sie keine Priester sind. Priester wird der Medizinmann, sobald er aus der Religion sein (ausschließliches) Gercerbe macht. Ein Medizinmann unterscheidet sich nur durch sein übernatürliches Wissen von seinen Landsleuten, fischt und jagt aber wie sie usw.; vgl. F. MIÜLLER, Unter Tungusen und fakuten 114; JAnthr.I. XIX, 283, 293 ff.; XXIV: 91 f., 99, 128 f. 152; XXXVII, 167; Journ. Im. Folkl.4 XXI, 9 f.; Kenne, Man Past and Present 377; 49. Annual Report Americ. Bureau of Ethnology 431. Wie man weiB, sind auch dic Funktionen der Medizinmänner, Kähine und Shä'ir's dieselben. Für weitere Belege für die Identitat von Kahinen und 
bekannt gewesen sind, geht aus dem obigen ethnographischen Material $\mathrm{x}$ ) hervor, nicht minder aus der unzweifelhaft historischen Mitteilung ${ }^{2}$ ), Muhammed habe in dieser ersten Periode geglaubt, er sei Kähin geworden 3), und aus dem Umstand, daß in Sürah 8I, 19-26, unmittelbar nach der Beschreibung der Vision, die Versicherung folgt: M. ist kein Kähin.-

Dies alles scheint mir, nach dem, was wir im ersten Kapitel bemerkt haben, einen genügenden Beweis dafür zu liefern, daß die Vorstellung der Brusteröffnung, verbunden mit der Himmelfahrt Muhammeds, aus dem altarabischen Gedankenkreis entlehnt worden ist.

IV.

Im Koràn finden wir, wie wir oben dargestellt haben, in Sürah 53 eine Erzählung über die initiierenden Träume, die M. gehabt hat, und so stellt diese Sūrah den historischen Bericht über den Beginn der Offenbarung dar. Lesen wir sie genau, so fällt wieder, wie stets, das Fragmentarische auf, das wir auch aus den anderen koranischen Erzählungen kennen. Muhammed (oder Allah!) scheint auf gewisse der Zuhörerschaft bekannte Dinge anzuspielen. Aus einer späteren Periode von Muhammeds Leben wissen wir, wie er die Autorität Allahs benutzte, nur wenn seine eigene nicht hinreichte (vgl. SNouckHurgronje, Gids I886, II 269). Dazu kommt, daß M. (Allah) immer Bezug auf das nimmt, was die Mekkaner über den Gegenstand sagen. Den verspotteten Propheten tröstet und schützt das Wort Allahs! Die Mekkaner kennen jedoch den Inhalt der Offenbarung schon im voraus; sonst könnte "Allah« ihre Meinung nicht tadeln. Dies setzt also voraus, daß M. selbst die Sachen zuvor den Leuten mitgeteilt hat und der.. Koran auf vorher Erzähltes anspielt. Wir haben

Medizinmännern vgl. Lammens op. cit. 257 mit MikHailovskir, JAnthr.I. 1895 (XXIV); 129 f., 144, 150, 155. Die Kähine bleiben noch während des Islam Lammens, ibid. Mirhailovskil 65, 92, 131, $138 \mathrm{f}$. Vgl. noch Hunrt, Histoire des Arabes I $98 \mathrm{f}$.

Man vergleiche noch SPENCER, Principles of Sociology VI 709: It should be added that the medicine man proper has some capacity for higher development as a social factor... sometimes (conducing) to the attainment and maintenance of poli tical power.

Auch die hebräischen Propheten sind wohl (Vier kandr, Die Stetigkeit im Kulturwandel $38 \mathrm{ff}$.) aus ähnlichen Medizinmännern hervorgegangen (FRAzER, Golden Bough 3 IV 66 ff.). Vgl. JAnthr.I XXIV, 99. Darum steht auch ihrer Initiation die des M. am năchsten, oben S. 21. Vgl. noch Horovitz, Islam $V_{48 .}$

3) Oben S. 2 bis 5 .

2) Diese Parallele zwischen M. und den Kähinen usw. ist historisch sehr bedeutsam, da M. öfters bezeugt, er sei kein Kähin usw., und man die Ähnlichkeit abzustreiten sucht, z. B. Sürah $26: 210,221 \mathrm{ff}$.

3) Caetani, Annali I 201; Goldzher, Abhandlungen I 5. Die Parallele wäre noch weiter durchzuführen gewesen; vgl. Caetani, Annali 1. c. 
gesehen, wie Brusteröffnung und Himmelfahrt $h$ is to $r$ is $\mathrm{ch}{ }^{1}$ ) zusammen gehören. Im Koran finden wir von der ersteren nichts 2$)$, sondern nur die zweite erwähnt 3). Wir müssen also schließen, daß Allah nur dasjenige hervorhebt, was er momentan betonen will. In 53 wie in 83 ist die Absicht, zu behaupten, M. erhalte seine Offenbarungen nicht von einem Diinn, sondern von Allah selbst, den er zweimal zu schen geglaubt hat: also kann die Brusteröffnung, die für die Mekkaner nichts Ungewöhnliches war, weil sich die ganze Geschichte an die KähinVorstellungen anschließt, ruhig wegbleiben. Nicht die Einzelheiten des Erlebnisses sind die Hauptsache, sondern der Autor, d. h. Alla h, nicht ein Djinn (Sūrah 26: 193f., 210). -

Neben der authentischen Erzählung in Sürah 53 über den Anfang der Offenbarung finden wir noch zwei andere Geschichten:

I. Die Traditionen, welche die Priorität von Sürah 96, I-5 behaupten, aber gar keinen Anhaltspunkt im Koran haben, obgleich 96, I ff. sehr gut eins der ersten Koranstücke sein kann 4).

Wie schon z. B. Bevan 5) bemerkt hat, verrät diese Überlieferung dem Sprachgebrauche nach die Beeinflussung von $\mathcal{F}$ es. to : 6 . Die Lokalisierung dieses Ereignisses auf Hirā' hat die Tendenz, die altarabische Heiligkeit diescs Berges für den Islam zu wahren ${ }^{6}$ ).

2. Die Überhieferungen, welche 74 , I als erste Offenbarung:) betrachten und nichts enthalten als eine Ausschmückung ${ }^{8}$ ) von Sürah 53, I-IO, verbunden mit dem Bericht über M.s epileptische Anfälle, die das Heryorheben von 74, I ff. wohl veranlaßt haben 9) ro).

Nachdem I. vom Idjmă als richtig anerkannt war, erfand man die Fatrah, um die Differenz auszugleichen. Wir können den Prozeß bei Muslim ${ }^{11}$ ) noch genau verfolgen. Erst schaltet man listig nach تئ رسو ألم

2) Im Sinne von WobBermin, Geschichte und schaft. Vgl. oben S. 6 ff. und Hartmann, Türk. Bibl. Bd. XVIII 157 Z. $15 \mathrm{ff}$.

2) Oben S. If.

3) Oben S. 14 Anm. I.

4) Snouck-Hurgronje, RHR XXX 155, Nöldeke-Schwally 78.

5) Bei Nicholson, Litterary History of the Arabs 151 Anm. I.

6) Snouck-Hurgronje, Mekka II 321; vgl. 319. Het Mekkaansche Feest 181 .

7) Vgl. das Ich-Stück in der Tradition T ab., Annales I 1155.

8) $\mathrm{DaB}$ dies richtig ist, erhellt sofort, wenn man die andere Variation in Betracht zieht, die Sūrah 53, I ff. zum ältesten Stück des Korans zu machen scheint ( $T$ ab. Tafs. XXVII 24).

9) Vgl. NöLdeke-Schwally I 87.

10) Man könnte nun vielleicht auch denken, daß unsere Erzăhlung eine Ausschmūckung von Sürah 53, I3 ff. sei. Ein triftiger Beweis dagegen ist die von der Ethnologie festgesetzte Untrennbarkeit der Brusteröffnung und Himmelfahrt.

ix) Muslim I 57 (Tab. Ann. I 1155 infra). 


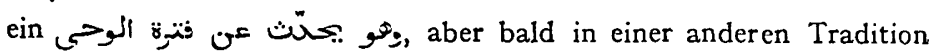

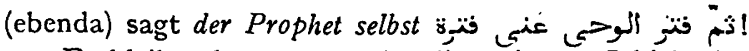

Es bleibt also noch übrig, die weiteren Schicksale zu skizzieren, die die Himmelfahrt in späterer Zeit erfahren hat. Vielleicht ist es nicht zu kühn, zu vermuten, daß eben das Anknüpfen unserer Erzählung an parallele Erscheinungen bei den Kähinen mitgewirkt hat, die wirkliche Bedeutung der Himmelsreise zu verdunkeln ${ }^{\mathrm{x}}$ ). Jedenfalls hat die übereinstimmende Meinung der muslimischen Gemeinde sie verleugnet und die Überlieferung, die die Priorität von Sūrah 96, I ff. hervorhebt, als die richtige erkannt. Die Ausbildung des $M i^{`} r a \bar{d} j$ nach dem Muster der alten christlichen u. a. ekstatischen Himmelreisen, die exzerptartige Mitteilung in Sürah 53, wo die Brusteröffnung fehlte, das nicht mehr Verstehen (-wollen und -können) der wirklichen Tendenz der Geschichte, die Verbindung mit der Nachtreise, die Fassung der Legende als Verklärung Muhammeds (oben S. I 5), dies alles und der Umstand, daß die Brusteröffnung an sich schon initiierende Bedeutung hat (oben S. 6), haben die enge Verbindung von Brusteröffnung und Himmelfahrt gelöst. Noch lange behaup. tete die erste ihre initiierende Bedeutung, wie u. a. aus einer Tradition bei Tabarīi ) und einer Mitteilung von Ibn Sajjid al-Nās 3) hervorgeht 4), welch letzterer sie mit der Ikra'-Tradition verknüpft!

Es scheint, daß neben der gewöhnlichen Erklärung von Sūrah 94, I auch eine weniger symbolische bestanden hat, die gleichfalls in gewisser Weise auf eine Art erlebter Weihe hinwies 5). Dies erleichterte wahrscheinlich die Verschmelzung der beiden. Weil die Autorität der Ikra'-Tradition immer wächst, verliert die Brusteröffnung ihre initiierende Bedeutung, obgleich sie später noch unter. den 'alämät

3) Man beachte die ziemlich kurze Zeit, in der der Prozeß sich vollzogen hat. Schon bei Ibn Hishãm und Ibn $\mathrm{Sa}_{a}$ dd ist die Himmelfahrt völlig legendär geworden, hat der Idjma $\bar{a}^{-}$das selbständige Bestehen der aus einer tendenziösen Erklärung erwachsenen Nachtreise nach Jerusalem (isr $\vec{a}$ ) anerkannt, ist die Brusteröffnung in die Jugendperiode verlegt, und hat man den einweihenden Charakter des Ganzen verkannt.

2) Annales I $1154 \mathrm{f}$.

3) Sprenger, Leben Mohammeds I 167 f.; vgl. noch Zotenberg, Tabarī II 24 I.

4) Hier sieht man wieder eine parallele Entwicklung zu SNouck-HUrgron JE, Mahdi 16 (Separatabdruck).

5) Tab. Tafs. XXVII 26 supra; vgl. Od. Salom. XI und Weiner, 1. c. 92 und eine Bemerkung desselben (212 Anm. 3):

1... Man hat mit Recht darauf hingewiesen, daß ein Symbol für jene Zeit etwas anderes ist als für uns: für uns ist es ein sinnliches Ding, das einen unsichtbaren Gegenstand darstellt, aber nicht enthält: für jene Zeit fällt in irgendeinem Sinne beides zusammen.* 
al-nubueveal genannt wird 1$)$. Auch ihr ursprünglicher Charakter wird verkannt [vgl. unsre Schlußbemcrkung zum I. Kapitel (oben S. 10)]. Man betont vielmehr die Reinigung in der Erzählung und beginnt zu reden von dem Teil des Teufels, der weggenommen wird 2). Die Figur Muhammeds soll ausgeschmückt werden! Erst wird er als sündenfrei betrachtet, als er als Prophet auftritt; später, als der Islam im Wettbewerb dem Christentum gegenüber ein "Evangelium Infantiae " braucht, soll er schon in seiner Jugend sündlos gewesen sein 3): die Geschichte wird in die Fugend verlegt: dies geschah um so leichter, als die Zeitangabe inzwischen verloren gegangen war 4). So wird die Erzählung ein fester Zug in den Jugendlegenden und, weil man einen Anhaltspunkt für ihn im Koran suchte, in späterer Zeit auch 5) eine materialisierende Erklärung von Sürah 94, I. Im ersteren Falle wird sie meist mit der wahrscheinlich aus jüdischen Kreisen ${ }^{6}$ ) hervorgegangenen Geschichte vom Wiegen verbunden, die bald vor 7 ), bald nach ihr ${ }^{8}$ ) erzählt wird.

Mit der eigentlichen Himmelfahrt war es nicht besser bestellt. Weil jede Chronologie in dieser 9) Weise verloren gegangen war, konnte man sie ohne Will kür nicht recht in der Muhammedbiographie eingliedern ${ }^{10}$ ), wenn man sie nicht, wie man öfters tut, mit der Nachtreise verband.

Leiden, Anfang April I9r4.

Nàchschrift während der Korrektur.

Dieser Aufsatz war schon längst dem Herrn Redakteur zugeschickt, als Herr Prof. Dr. A. J. Wensinck (Leiden) wieder die außerordentliche Freundlichkeit hatte, meine Aufmerksamkeit auf den Artikel Bevans in den "Studien zur semitischen Philologie und Religionsgeschichte, Juluus Wellhausen zum siebzigsten Geburtstag am

3) Ibn Sa‘d I 70 und DãrimI (Leidener Handschr., folio 4); Baghawi II 176; Mishkät (ed. 1268) 516.

2) Z. B. Tab. Ann. I II55 u. a. S.

3) Zotenberg, Tabari II 241.

4) Tabari, Aní. 1. c.

5) Selbst noch nicht bei Baghawi im Ta/sir (Leid. Handschr.); vgl. oben S. 5; diese Erklärung findet sich erst bei Baidăwi zu 94, 1. Vgl. noch MuIR, Life I 21 infra.

6) Wie die Geschichte der Niederkunft: WEIL, Biblische Legenden uș. 129 Anm., 133.

7) T ab. Ann. l. c.

8) Mas'ūdi IV I3I; die Geschichte steht ganz auf sich selbst bei Därimì (Leid. Handschr.) folio 5 a.

9) Vgl. jedoch Caetani, Annali I 229.

so) Vgl. oben S. 5, 15; vgl. noch Ba ghawi Tafsir (Leid. Handschr.) III Folio I 
17. Mai I9I4 gewidmet ... herausgegeben von KaRL MARTI« über Mohammeds Ascension to Heaven zu lenken. Ich freue mich sehr, daß die Ergebnisse meiner Untersuchungen in vielem mit den seinigen übereinstimmen. Auch er sagt: ఎThe story of the Ascension ... carries us back to the very beginning of Mohammed's prophetic career» (S. 56). Er weist auf die Wichtigkeit der Angabe Tabarī's hin, der in seinen Annales (oben S. 5) dies ausdrücklich behauptet, indem va statement in the Chronicle represents the author's final verdict after a full consideration of the evidence which he had before him" (S. 57). Obweil seiner Ansicht nach die Brusteröffnung "was suggested by. chap. 94: I of the Koran " (S. 58), eine Behauptung, die, wie oben gezeigt, nicht richtig ist, hält er doch Brusteröffnung und Himmelfahrt zusammen. "The cleansing ceremony forms a natural introduction to Mohammed's prophetic career. Consequently when the Ascension was shifted from its original position and placed at a later period, the cleansing was no longer an appropriate feature (S. 58).

Zum Schluß möchte ich den Herren Professoren Dr. C. H. Becker (Bonn), Dr. C. Snouck Hurgronje (Leiden) und Dr. S. R. Steinmetz (Amsterdam) meinen herzlichen Dank bezeugen für manche bereichernde oder anregende Bemerkung. Dr. E. GraÉFE (Hamburg) hatte die außerordentliche Freundlichkeit, das Deutsch meines Aufsatzes durchzusehen, wodurch ich auch ihm zu aufrichtigstem Danke verpflichtet bin.

Ende Juli I9I4.

Anmerkung. Zu Seite 5 Anm. 2. Nachdem Baghaw I (Tafsïr Leid. Handschr.

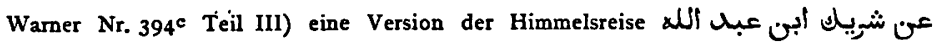

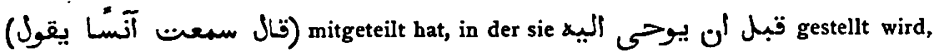
sagt er : (Folio $3^{\mathrm{b}}$ infra)

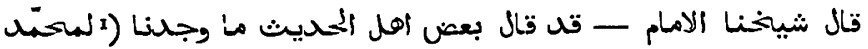

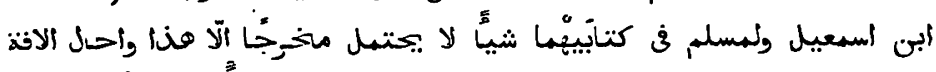

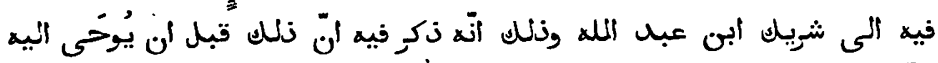

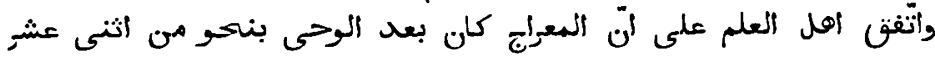

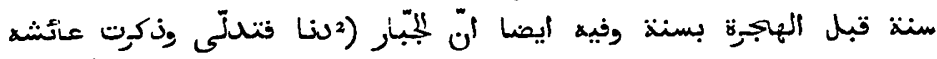

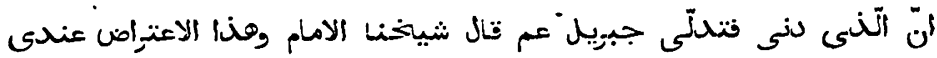

1) Bu c b a ri i.

3) Sürah $53: 8$. 


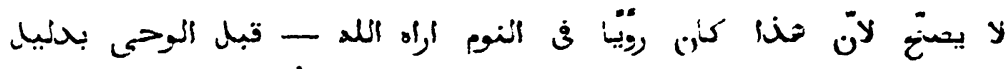

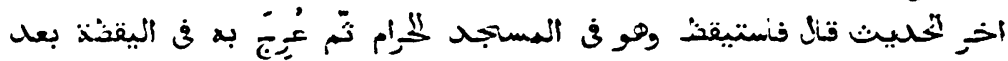

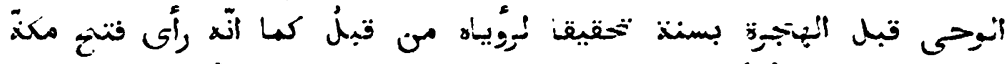

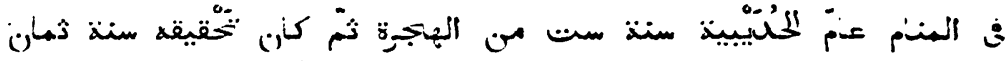

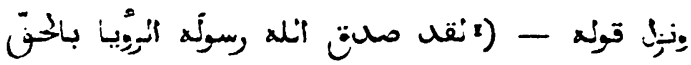

3) Sürah 43:27. 